\title{
Direct High Resolution Cryo-TEM Imaging of Liquid Crystals
}

\author{
M. Gao ${ }^{1}$, C. Zhang ${ }^{2}$, H.S. Park ${ }^{1}$, A. Jákli ${ }^{1,2}$ and O.D. Lavrentovich ${ }^{1,2}$ \\ ${ }^{1 .}$ Liquid Crystal Institute, Kent State University, Ohio, USA \\ 2. Chemical Physics Interdisciplinary Program, Kent State University, Ohio, USA
}

Liquid crystals represent a group of materials with tremendous scientific and technological importance. However, they also impose great challenges on direct electron microscopy imaging due to the unparalleled irradiation damages under electron beam and the lack of effective specimen preparation methods. As a result, a replica transmission electron microscopy (TEM) technique, namely freeze fracture TEM, has been the dominant nanoscale imaging tool [1]. Due to the limitations of the size of the deposited metallic nano-clusters and the correspondence between the fractured surface and the native structures, a sub-nanometer resolution is very difficult, if not impossible, to be achieved using such replica technique. In this paper, we present our initial efforts towards high resolution direct TEM imaging of liquid crystals, which may greatly enhance those studies on phase transitions and defects at near molecular scale, interactions and interfaces between liquid crystals and nanoparticles, etc.

Liquid crystals undergo different phases (e.g., isotropic, nematic, smectic, and crystalline) as the disordered molecules gain orientational and/or positional orders. In this study, we focus on the observation of smectic domains in both thermotropic and lyotropic materials. A modified cryo-TEM low-dose procedure has been developed to locate and image the areas of interest (e.g., layered smectic clusters). A representative dose of $20 \mathrm{e}^{-} / \mathrm{nm}^{2}$ has been used in searching mode, and $200 \mathrm{e}^{-} / \mathrm{nm}^{2}$ in recording mode to minimize the irradiation damage, which are 1-2 orders lower compared to those used in routine cryo-TEM observation of biomaterials. We demonstrate that a resolution better than $0.7 \mathrm{~nm}$ can still be obtained at such weak electron dose. An effective specimen preparation procedure has also been developed for thermotropic liquid crystals, enabling deposition of electron transparent liquid crystal thin films followed by thermal processing and rapid plunge freezing in liquid nitrogen or liquid ethane. Figure 1(a) shows a typical cryo-TEM image and the corresponding FFT pattern of a long-range layered smectic structure in a bent-core liquid crystal quenched at $87^{\circ} \mathrm{C}$, indicating a well-defined period of $3.7 \mathrm{~nm}$. The periodic structure can remain aligned in domains larger than 1 micron, and the direction of the alignment can change smoothly inside the domains. Figure 2 shows the first direct evidence of smectic clusters (periodicity ranging between $3.1 \mathrm{~nm}$ and $3.5 \mathrm{~nm}$ ) in a nematic liquid crystal [2]. The structures observed at different quenching temperatures clearly show the increasing number and size of the smectic domains as the quenching temperature decreases [2]. Similar observation can be performed to lyotropic liquid crystals by combining the abovementioned cryo-TEM procedure and routine plunge freezing. Figure 1(b) shows a typical image of the observed smectic layers. The alignment direction of the layered structure remains relatively uniform inside each domain. In addition, some liquid crystals exhibit strong thickness effect, i.e., the native structure cannot be preserved in thin film specimens. To overcome the challenge, we have employed high pressure freezing and cryo-ultramicrotomy, and initial success has been achieved in lyotropic liquid crystals.

By probing the liquid crystals directly, many TEM techniques beyond traditional imaging become possible, for example, scanning TEM (STEM) Z-contrast imaging, energy-dispersive x-ray spectroscopy, and electron diffraction. Figure 1(c) shows a Z-contrast image of gold nanoparticles dispersed in a thermotropic liquid crystal. The Z-contrast imaging can greatly enhance the contrast and 
visibility of the nanoparticles especially in thick areas. Part of the techniques developed in this study can also be applied to other soft-matter materials [3].

References:

[1] L.E. Hough, et al, Science 325 (2009), 456.

[2] C. Zhang, et al, Phys. Rev. Lett. 109 (2012), 107802.

[3] The TEM data were obtained at the cryo-TEM facility at the Liquid Crystal Institute, Kent State University, supported by the Ohio Research Scholars Program. The authors thank Drs. W. Weissflog and U. Baumeister for providing liquid crystal samples, and Drs. S. Sprunt and J.T. Gleeson for helpful discussions.
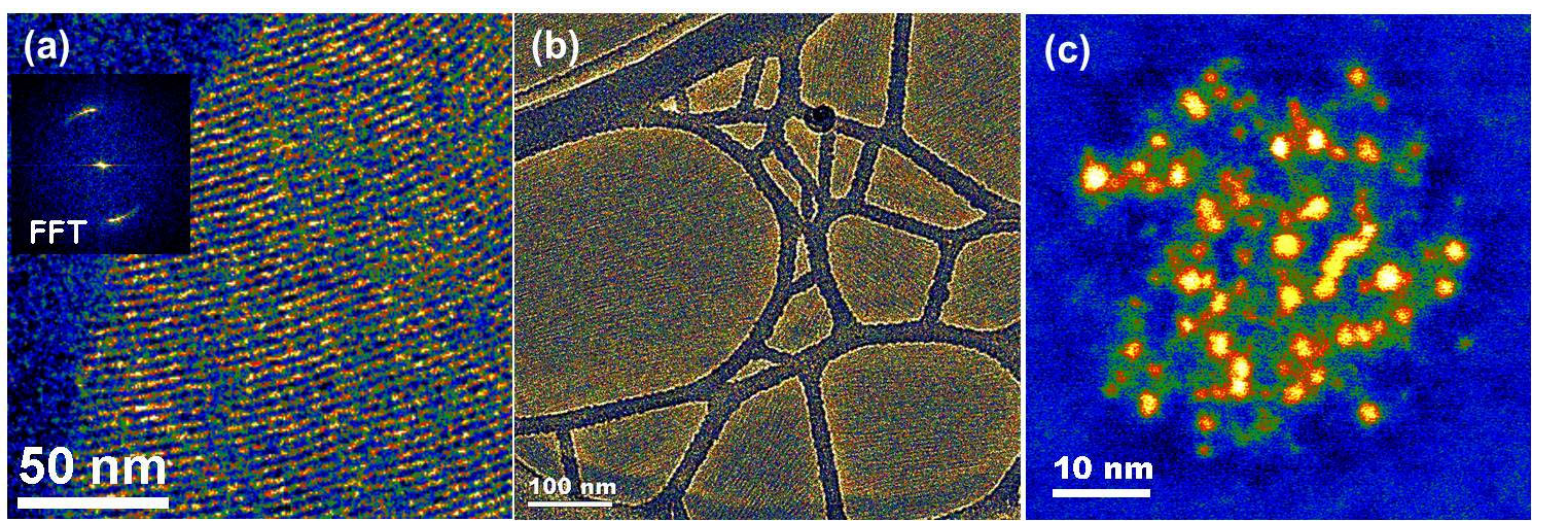

Figure 1. (a) Cryo-TEM image of a thermotropic liquid crystal quenched at $87^{\circ} \mathrm{C}$. The inset shows the corresponding FFT pattern. (b) Cryo-TEM image of a lyotropic liquid crystal quenched at room temperature. (c) Cryo-(S)TEM Z-contrast image of gold nanoparticles dispersed in a thermotropic liquid crystal.

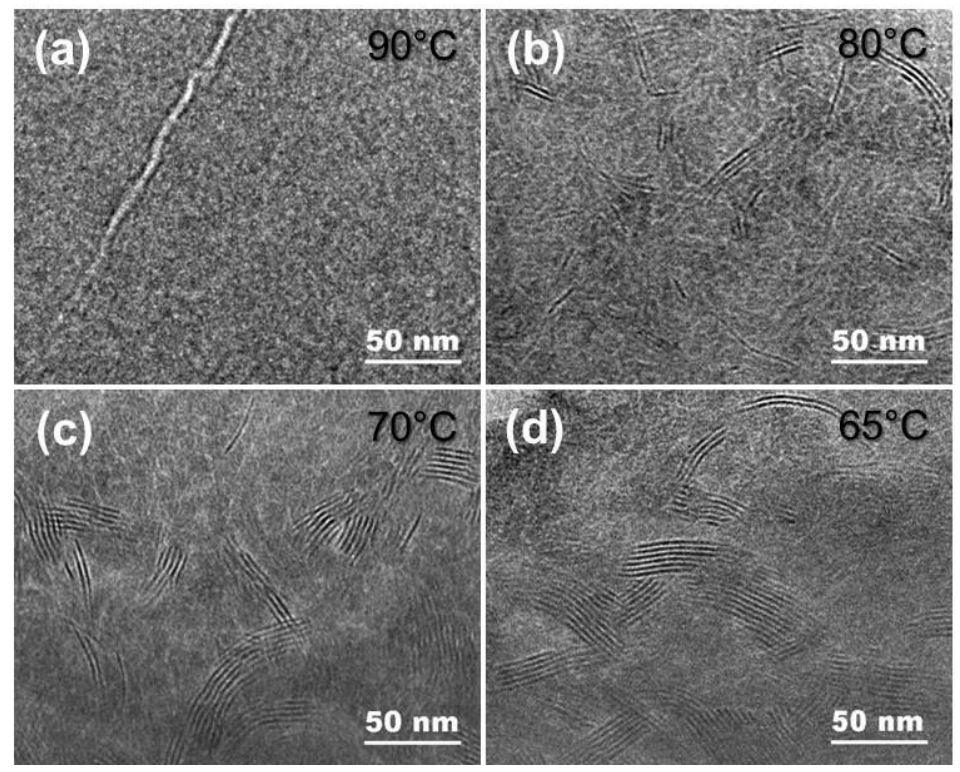

Figure 2. Cryo-TEM images of a thermotropic liquid crystal, showing the quenched structures as a function of the quenching temperature. 